\title{
Renal artery stenosis of a solitary kidney with successful salvage of unusual complication of renal artery angioplasty
}

\begin{abstract}
Renovascular disease is an important, potentially correctable cause of secondary hypertension. The frequency with which it occurs is variable. It accounts for less than 1 percent of cases of mild to moderate elevations in blood pressure. ${ }^{1}$ In contrast, the prevalence is much higher in patients with acute (even if superimposed upon a preexisting elevation in blood pressure), severe, or refractory hypertension. ${ }^{2}$ Several options exist for the treatment of renovascular hypertension: medical management, surgical revascularization, or percutaneous transluminal renal artery angioplasty (PTRA). ${ }^{3,4}$ We reported a 19-yearold male patient who was suffering from severe hypertension which was not controlled despite 5 antihypertensive drugs. Routine investigations revealed high serum creatinine $7.3 \mathrm{mg} / \mathrm{dl}$, serum $\mathrm{K} ; 2.8 \mathrm{meq} / \mathrm{L}$ with normal other lab results. The patient had manifestations highly suggestive of pulmonary edema, so hemodialysis with ultrafiltration had been instituted and the patient condition had improved. Further investigations revealed renal artery stenosis of right solitary kidney. So, right renal angioplasty with stenting had been decided. However, the procedure complicated with broken guide catheter tip in the aorta and the renal artery. Immediate abdominal exploration was carried out for removal of the broken part. The surgery passed smoothly and the patient referred to ICU with stable hemodynamics. Another trial for right renal angioplasty was attempted with successful fixation of two stents and the patient blood pressure and serum creatinine had improved The patient discharged with normal kidney function. PTRA is considered one of the most important methods for renovascular hypertension treatment with successful results. The technical complication rate is between 5 and 15 percent. ${ }^{1,5}$ Most of the complications are relatively minor and include puncture site hematoma and renal artery dissection. However, more serious problems can arise like what we reported.
\end{abstract}

Keywords: renal artery stenosis, PTRA, hypertension, renovascular disease.
Volume 6 Issue 4 - 2018

Yasser Elsayed Matter,' Hussein Attia

Sheashaa,' Ahmed Yahia Elmowafy',

Mohammed Ashraf Fouda,' Ayman Fathi

Refaie,' 'Tarek Medhat Abbas,' Mohamed

Hamed Abbas,' Muhammed Ahmed Elhadedy'

Tarek El-Diasty ${ }^{2}$

'Department of Dialysis and Transplantation, The UrologyNephrology Center, Mansoura University, Egypt

${ }^{2}$ Department of Radiology, The Urology-Nephrology Center,

Mansoura University, Egypt

Correspondence: Yasser Elsayed Matter, Urology \& Nephrology Center, Mansoura University, P.O.Box:35516, Egypt, Tel 0020502202222, Fax 0020502263717,

Email yassermatter86@gmail.com

Received: May 29, 2018 | Published: July 23, 2018
Abbreviations: RVH, renovascular hypertension; RAS, renal artery stenosis; HTN, hypertension; HD, hemodialysis; PSV, peak systolic velocity; ACE, angiotensin-converting enzyme; ARB, inhibitor or angiotensin II receptor blocker; ASTRAL, Angioplasty and Stenting for Renal Artery Lesions; CORAL, Cardiovascular Outcomes in Renal Atherosclerotic Lesions

\section{Introduction}

Renovascular disease with reduced perfusion of the kidney can produce a range of clinical syndromes, most frequently a rise in arterial pressure, renovascular hypertension (RVH), with or without associated ischemic and hypertensive renal injury. RVH is usually caused by renal artery stenosis (RAS) and is a common secondary form of hypertension (HTN). ${ }^{6}$ PTRA has become an increasingly widespread peripheral vascular intervention for the treatment of the RVH. Alone or in combination with stent implantation, PTRA is increasingly used as an alternative to surgical revascularization for the treatment of RAS, which may cause HTN or jeopardize renal function. Technical success is usually achieved in more than $85 \%$ of cases; the failure rate is $10 \% .^{7-9}$ To our knowledge, this is the first time to report PTRA complication of a broken guide catheter tip in the aorta and the renal artery with successful salvage of the renal artery in the literature.

\section{Case report}

A 19- year- old male patient who was suffering from uncontrolled hypertension despite 5 anti-hypertensive drugs. Routine investigations revealed high serum creatinine $7.3 \mathrm{mg} / \mathrm{dl}$, serum $\mathrm{K} ; 2.8 \mathrm{meq} / \mathrm{L}$ with normal other lab results. The patient urine output was approximately $1000 \mathrm{ml}$ per day. On examination, blood pressure of 240/110, bilateral midzonal and basal crepitation of chest, mild bilateral lower limb edema, respiratory rate of 32/minute and hypoxia (So2 was $85 \%$ ) were reported. Therefore, the patient received oxygen support and hemodialysis (HD) session with ultrafiltration of 4 liters on a provisional diagnosis of pulmonary edema with improvement of the patient status. Renal ultrasound showed small sized atrophic pyelonephritic left kidney and the right kidney size was $12.5 * 4 \mathrm{~cm}$ with normal sonoghraphic appearance. Renal Doppler showed absent diastole in most of waves of the right renal vessels, resistive index (RI) of right main renal artery was 0.82 with peak systolic velocity (PSV) $22 \mathrm{~cm} / \mathrm{s}$ and RI of right intrarenal artery was 0.87 with PSV $12.7 \mathrm{~cm} / \mathrm{s}$. Right renal artery stenosis was diagnosed and confirmed by computed tomography angiogram (CTA) which detected single right renal artery with narrowing of its caliber at the proximal part $(16 \mathrm{~mm})$ (Figure 1). Renogram had shown severe parenchymal tracer (MAG3) retention in the affected right kidney mostly due to renal artery stenosis and nonfunctioning left one. Thus, right renal angioplasty with stenting was 
decided. But, the procedure complicated with broken guide catheter tip (about $3 \mathrm{~cm}$ lengths) and the distal part was impacted in the renal artery and the proximal part in the aorta (Figure 2). Consequently, immediate abdominal exploration was done for removal of the broken part. The surgery passed smoothly and the patient referred to ICU with stable hemodynamics. The patient maintained on HD and hypertensive drugs for 3 weeks then the patient underwent a successful right renal angioplasty with two stents fixation (Figure 3). The blood pressure and serum creatinine started to improve, HD was stopped and the patient was discharged after 2 weeks with serum creatinine $0.9 \mathrm{mg} / \mathrm{dl}$, four antihypertensive drugs, warfarin and aspirin with cessation of warfarin six months later. Currently, the patient has maintained on 2 antihypertensive drugs, aspirin with stable kidney function and controlled blood pressure.

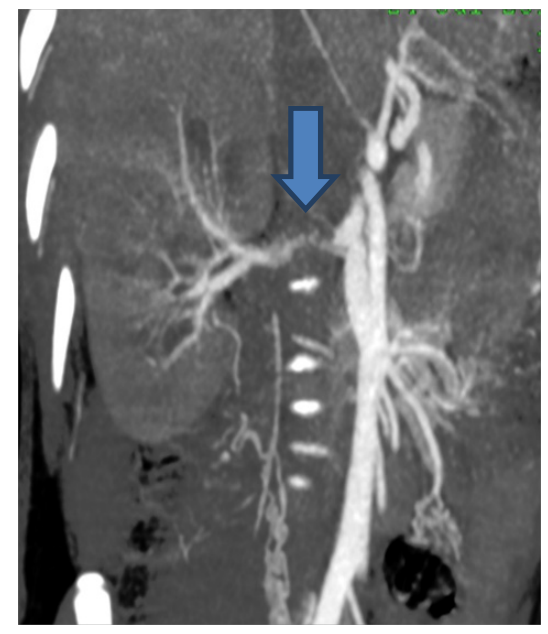

Figure I Computed tomography angiogram of renal artery demonstrates single right renal artery with narrowing of its caliber at the proximal part (the arrow).

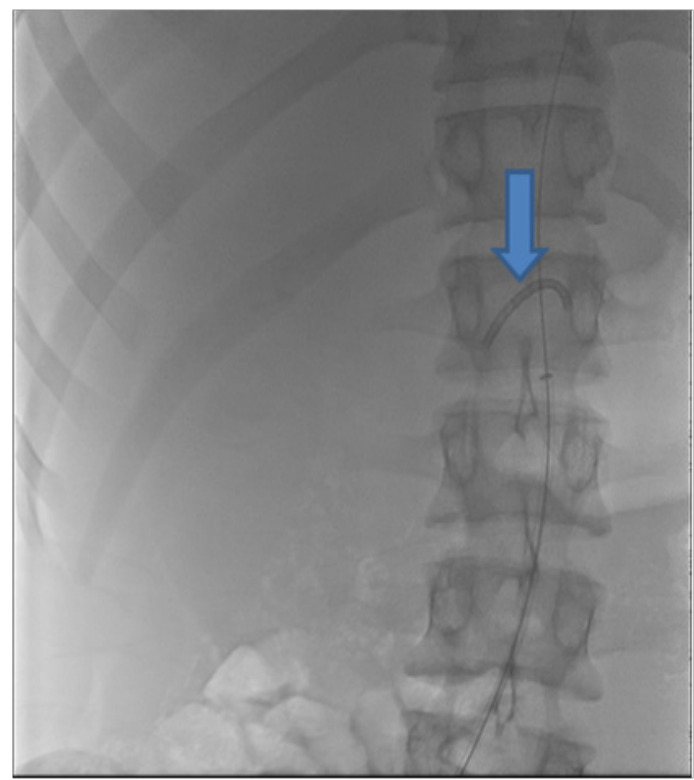

Figure 2 The broken guide catheter tip (about $3 \mathrm{~cm}$ length) with the distal end in the right renal artery and the proximal end in the aorta (the arrow).

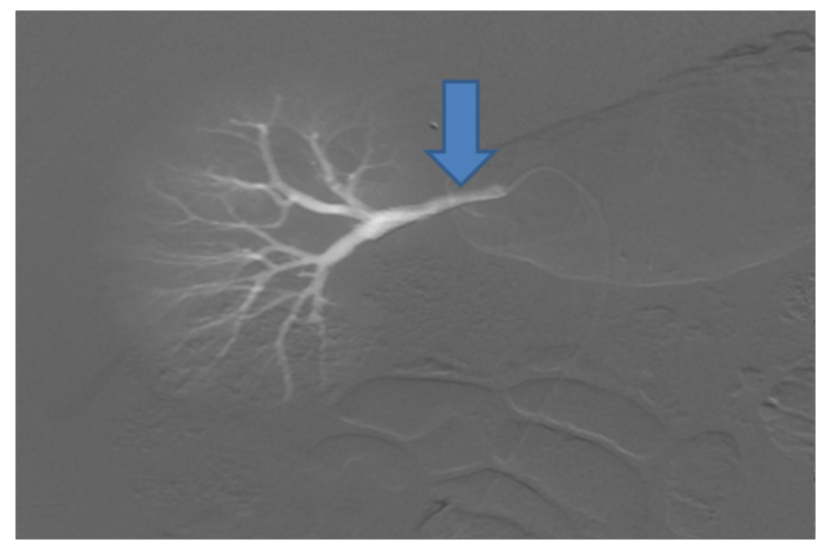

Figure 3 Successful right renal artery angioplasty and stenting (the arrow).

\section{Discussion}

PTRA with stenting is the preferred non-surgical intervention. We agree with the $2005 \mathrm{ACC} / \mathrm{AHA}$ guidelines on the management of peripheral artery disease, which recommended PTRA with stenting for ostial atherosclerotic lesions in patients who have clinical clues suggesting that the renovascular disease is likely to be responsible for hypertension that is not well controlled with optimal medical therapy.$^{10}$ Criteria suggest renovascular disease are :an acute elevation in serum creatinine of at least 30 percent after administration of angiotensin-converting enzyme (ACE) inhibitor or angiotensin II receptor blocker (ARB), moderate to severe hypertension in a patient with diffuse atherosclerosis, a unilateral small kidney, or asymmetry in renal size of more than $1.5 \mathrm{~cm}$ that cannot be explained by another reason ,moderate to severe hypertension in patients with recurrent episodes of flash pulmonary edema, Onset of stage II hypertension after age 55 years and systolic or diastolic abdominal bruit (not very sensitive). ${ }^{10}$ Many randomized clinical trials failed to verify cost-benefit from revascularization in patients with uncontrolled hypertension or impaired kidney function as ASTRAL (Angioplasty and Stenting for Renal Artery Lesions), CORAL (Cardiovascular Outcomes in Renal Atherosclerotic Lesions), and STAR (Stent Placement in Patients with Atherosclerotic Renal Artery Stenosis and Impaired Renal Function). ${ }^{11-13}$ In our case, the patient had severe resistant hypertension, high serum creatinine, solitary right kidney (the left kidney is atrophic and nonfunctioning) and pulmonary edema. The diagnosis of RAS is confirmed by CTA of the renal vessels. Based upon the available data, we recommend not performing PTRA with stenting in patients who do not meet the previous criteria because the likelihood of benefit is small and the risk of procedural complications is relatively high. The technical complication rate with PTRA with or without stenting is between 5 and 15 percent. Most of the complications are relatively minor and include hematoma at the puncture site and renal artery dissection. However, more serious problems can arise, including death, renal artery thrombosis or perforation (often requiring surgery) and acute renal failure due to atheroembolic disease or to a reaction to the radio contrast agent. ${ }^{1,5,14,15}$ In the largest trial, ASTRAL, included 806 patients with either unilateral or bilateral atherosclerotic renal artery stenosis who were randomly assigned to either medical therapy alone or medical therapy plus revascularization (95 percent with angioplasty with stent placement, 5 percent was angioplasty alone), there were 38 
complications that occurred within 24 hours of procedure in 31 of the 359 patients ( 9 percent) who underwent revascularization with stenting. Nineteen of these events were serious, including pulmonary edema and myocardial infarction; there were five renal embolizations, four renal arterial occlusions, four renal artery perforations, one femoral-artery aneurysm, and three patients with clinically significant cholesterol embolization. Among 280 patients who underwent revascularization and for whom data were available, 20 percent had an adverse event between 24 hours and one month. These included two deaths, four cases of groin hematoma or hemorrhage, five cases of acute kidney injury, and one renal artery occlusion. ${ }^{5}$ Interestingly, the interventional radiologist was the same person in the two PTRA procedures (the last author). Since five years and till now, the patient shows stable kidney function and controlled blood pressure with only two antihypertensive drugs. In conclusion, because of the potential for harm from invasive procedures, PTRA should be considered for patients who are thought to have a high likelihood of benefiting from the procedure in presence of the previous mentioned criteria. Also, PTRA should be done in experienced and well equipped centers.

\section{Acknowledgements}

I want to thank all the authors for their valuable effort in this manuscript.

\section{Conflict of interest}

The authors declare that they have no conflict of interest.

\section{References}

1. Dworkin LD, Cooper CJ. Renal-artery stenosis. $N$ Engl J Med. 2009;361(20):1972-1978.

2. Textor SC, Lerman L. Renovascular hypertension and ischemic nephropathy. Am J Hypertens. 2010;23(11):1159-1169.

3. Aristizabal D, Frohlich ED. Hypertension due to renal arterial disease. Heart Dis Stroke. 1992;1(4):227-234.

4. Detection, evaluation, and treatment of renovascular hypertension. Final report. Working Group on Renovascular Hypertension. Arch intern Med. 1987;147(5):820-829.

5. Astral Investigators, Wheatley $\mathrm{K}$, Ives $\mathrm{N}$, et al. Revascularization versus medical therapy for renal-artery stenosis. $N$ Engl $j \mathrm{Med}$. 2009;361(20):1953-1962.

6. Richard J Johnson, John Feehally, Jürgen Floege. Comprehensive clinical nephrology. 5th ed. Philadelphia: Elsevier; 2015. 1320p.

7. Jenks S, Yeoh SE, Conway BR. Balloon angioplasty, with and without stenting, versus medical therapy for hypertensive patients with renal artery stenosis. Cochrane Database Syst Rev. 2014;12:CD002944.

8. Herrmann SM, Saad A, Textor SC. Management of atherosclerotic renovascular disease after Cardiovascular Outcomes in Renal Atherosclerotic Lesions (CORAL). Nephrol Dial Transplant. 2015;30(3):366-375.

9. Textor SC, McKusick MM. Renal artery stenosis: if and when to intervene. Curr Opin Nephrol Hypertens. 2016;25(2):144-151.

10. Hirsch AT, Haskal ZJ, Hertzer NR, et al. ACC/AHA 2005 Practice Guidelines for the management of patients with peripheral arterial disease (lower extremity, renal, mesenteric, and abdominal aortic). Circulation. 2006;113(11):e463-e654.

11. Bax L, Woittiez AJ, Kouwenberg HJ, et al. Stent placement in patients with atherosclerotic renal artery stenosis and impaired renal function: a randomized trial. Ann Intern Med. 2009;150(12):840-848.

12. Cooper CJ, Murphy TP, Cutlip DE, et al. Stenting and medical therapy for atherosclerotic renal-artery stenosis. $N$ Engl $J$ Med. 2014;370(1):13-22.

13. ASTRAL Investigators, Wheatley $\mathrm{K}$, Ives $\mathrm{N}$, et al. Revascularization versus medical therapy for renal-artery stenosis. $\mathrm{N}$ Engl $\mathrm{J}$ Med. 2009;361(20):1953-1962.

14. Rocha Singh K, Jaff M R, Rosenfield K, et al. Evaluation of the safety and effectiveness of renal artery stenting after unsuccessful balloon angioplasty: the ASPIRE-2 study. $J$ Am Coll Cardiol. 2005;46(5):776-783.

15. Blum U, Krumme B, Flügel $P$, et al. Treatment of ostial renal-artery stenoses with vascular endoprostheses after unsuccessful balloon angioplasty. $N$ Engl J Med. 1977;336(7):459-465. 